Assessment of an ORION-based experimental platform for measuring the opacity of high-temperature and high-density plasma

P. Beiersdorfer

September 10, 2012 
This document was prepared as an account of work sponsored by an agency of the United States government. Neither the United States government nor Lawrence Livermore National Security, LLC, nor any of their employees makes any warranty, expressed or implied, or assumes any legal liability or responsibility for the accuracy, completeness, or usefulness of any information, apparatus, product, or process disclosed, or represents that its use would not infringe privately owned rights. Reference herein to any specific commercial product, process, or service by trade name, trademark, manufacturer, or otherwise does not necessarily constitute or imply its endorsement, recommendation, or favoring by the United States government or Lawrence Livermore National Security, LLC. The views and opinions of authors expressed herein do not necessarily state or reflect those of the United States government or Lawrence Livermore National Security, LLC, and shall not be used for advertising or product endorsement purposes.

This work performed under the auspices of the U.S. Department of Energy by Lawrence Livermore National Laboratory under Contract DE-AC52-07NA27344. 


\title{
Assessment of an ORION-based experimental platform for measuring the opacity of high-temperature and high-density plasma
}

\author{
Peter Beiersdorfer \\ Lawrence Livermore National Laboratory, Livermore, CA 94551
}

\begin{abstract}
The following provides an assessment of an experimental platform based on the ORION laser at AWE Aldermasten, England, for measuring the opacity of hightemperature and high-density LTE plasmas. The specific points addressed are (1) the range of electron density and temperature that can be achieved with short-pulse beams alone, as well as (2) by means of compression with a longpulse beam; (3) the accuracy with which electron density, electron temperature, and absolute emissivity can be measured; (4) the use of pulse shaping to increase the sample density to above solid density; (5) the effect that target materials and target design have on maintaining spatial uniformity of the sample, and (6) the need for additional diagnostics to produce and characterize samples for decisive measurements.
\end{abstract}

\section{Introduction:}

A sequence of novel experiments carried out by AWE and the Livermore Radiative Properties Group during the past four years on the HELEN laser at Aldermasten and the Titan laser at the Jupiter Laser Facility at Livermore have revealed the tantalizing possibility of measuring the opacity of various materials at high density and high temperature. The ORION laser at AWE was built with that specific goal in mind. In the first half of FY12, we have collaborated with AWE to assess ORION's capabilities and to determine whether they can be utilized for obtaining definitive opacity data in these novel plasma regimes.

\section{Current status of ORION:}

ORION was commissioned to fire 1- $\omega$ pulses late last year. Conversion to a 2$\omega$, green beam was accomplished in April 2012, although power levels are not yet at full design capacity.

Using the green beam, experiments have now been started to reproduce results from HELEN. The first results show that at a green beam energy of about 20 Joule, which is comparable to the energy achieved in the HELEN experiments, the ORION 
beam can reproduce the plasma parameters achieved by HELEN. This has validated the ORION laser and bodes very well that even better plasma parameters, i.e., higher electron temperature and higher electron density, will be achieved when the green beam fires at full capacity $(50-100 \mathrm{~J})$.

Moreover, the final ORION laser configuration will allow target compression with a long-pulse beam in order to achieve even higher densities, as shown in Fig. 1 below.

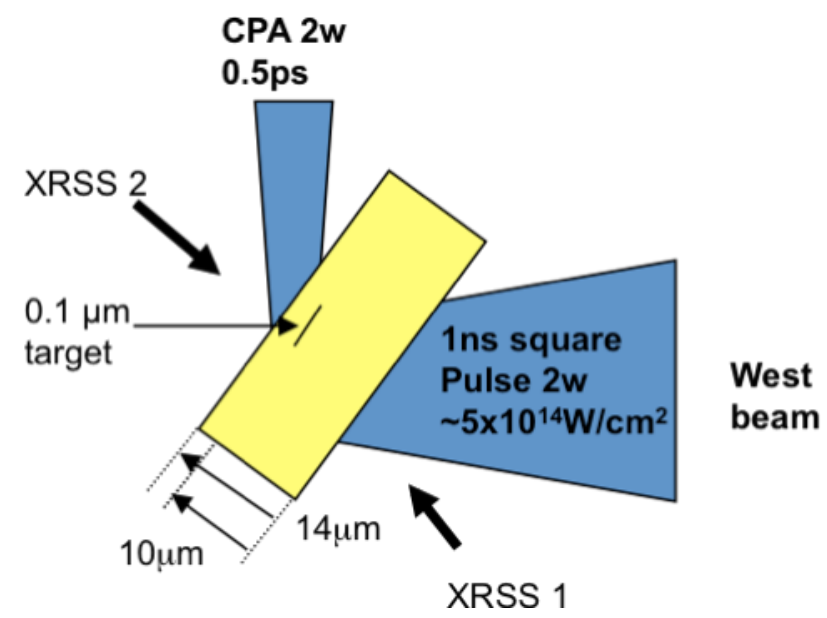

Fig. 1. Target arrangement for opacity measurements on ORION.

\section{Anticipated plasma parameters:}

The novel combination of high density and high temperature in opacity measurements requires several important technical steps, which are only now coming together in the right combination. The first is heating with a short pulse laser that has sufficient contrast so that no fast electrons are generated, which would change the ionization balance away from LTE. The contrast ratio of a typical 1- $\omega$ short-pulse beam is too low for this purpose. However, conversion to a 2- $\omega$ beam increases the contrast to an acceptable level. The 2- $\omega$ short-pulse beam on ORION now satisfies the contrast requirements, as demonstrated in April 2012.

Electron density and temperature: The plasmas achieved in April 2012 with ORION appear to match those achieved earlier with HELEN. The corresponding electron temperatures and densities are about 500 to $600 \mathrm{eV}$ and 1.0 to $1.5 \mathrm{~g} / \mathrm{cc}$. Based on these and previous results from HELEN and Titan, we make the following assessment of the temperature and densities achievable with the short-pulse beam alone. The assessment splits into three categories: parameters that are certain to be achieved, because they have already been achieved; parameters that are likely to be achieved, because ORION's design parameters have not yet been fully implemented so that we can expect further improvements; and parameters that can possibly be 
achieved, because optimizations may occur as opacity experiments are performed and new ways are explored. Our assessments are summarized in Table 1.

Increased density by compression: Long-pulse compression of the plasma was successfully demonstrated on HELEN. There, it had the effect of doubling the electron density. It is planned to use one to four long-pulse beams on ORION to compress the target. Because such experiments have not yet been carried out on ORION, we can only extrapolate from past experiments. We present our assessment of the plasma parameters (for an aluminum buried layer) that will result from longpulse beam compression in Table 1. As before, we use the same three levels of likelihood that a given plasma parameter will be achieved.

Table 1. Assessment of electron temperature and electron density that are expected to be achieved in ORION opacity experiments.

\begin{tabular}{|c|c|c|c|}
\hline \multicolumn{4}{|c|}{ Short-pulse beam alone } \\
\hline & Certain & Likely & Possibly \\
\hline $\begin{array}{l}\text { Electron } \\
\text { temperature }\end{array}$ & $500 \mathrm{eV}$ & $600-800 \mathrm{eV}$ & $1000 \mathrm{eV}$ \\
\hline $\begin{array}{c}\text { Electron } \\
\text { density }\end{array}$ & $1.0 \mathrm{~g} / \mathrm{cc}$ & $1.5-2.0 \mathrm{~g} / \mathrm{cc}$ & $1.5-2.0 \mathrm{~g} / \mathrm{cc}$ \\
\hline \multicolumn{4}{|c|}{ Short-pulse beam + long-pulse compression } \\
\hline & Certain & Likely & Possibly \\
\hline $\begin{array}{l}\text { Electron } \\
\text { temperature }\end{array}$ & $500 \mathrm{eV}$ & $600-800 \mathrm{eV}$ & $1000 \mathrm{eV}$ \\
\hline $\begin{array}{c}\text { Electron } \\
\text { density }\end{array}$ & $2.0-4.0 \mathrm{~g} / \mathrm{cc}$ & $3.0-6.0 \mathrm{~g} / \mathrm{cc}$ & $4.0-8.0 \mathrm{~g} / \mathrm{cc}$ \\
\hline
\end{tabular}

Increased density by compression with timed multiple beams: On ORION, it is also planned to adjust the timing of each beam to optimize compression. To assess this capability, we use guidance from modeling predictions because none of the earlier experiments have implemented multiple beams and pulse timing. Modeling predictions were provided to us by AWE and are shown in Fig. 2. These predictions indicate that four long-pulse beams can increase the target density by a factor of 10 , if appropriately timed, i.e., if the arrival time of the combined pulse is appropriately shaped. We think this is overly optimistic, as similar model predictions for HELEN experiments with one beam clearly over-predicted the observed compressed density. However, we conservatively assume in Table 1 that an increase by a factor of 4 is possible, albeit not likely. 


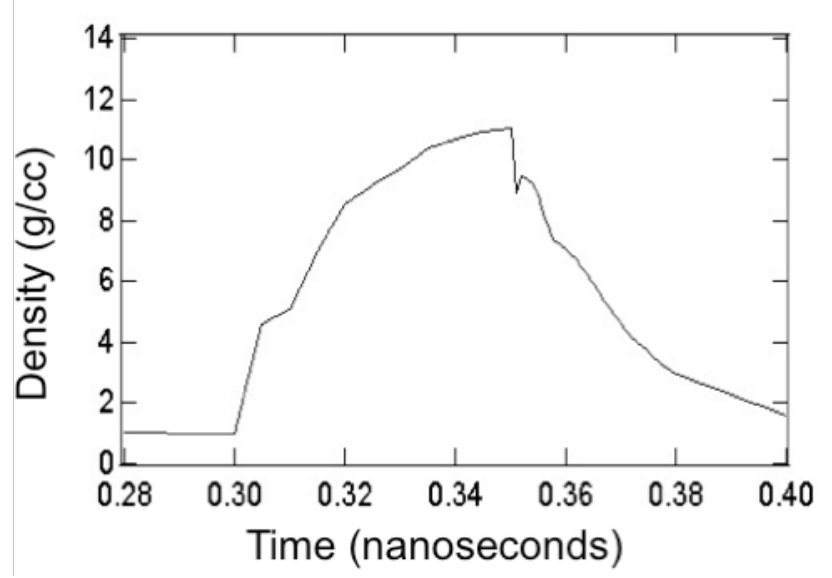

Fig. 2. Modeling predictions showing the expected target density as four long-pulse beams are timed to optimize target compression as a function of time.

Increased density achieved by target design choices: If the target density can be kept high before the compression beams arrive, it is likely that the compressed target density will be higher than if the target density has already blown down. This will be done by impedance matching of the tamper material to the buried layer material of interest. Past measurements relied on tamping the target material with plastic, which has a density of about $1 \mathrm{~g} / \mathrm{cc}$. Aluminum has a density of $2.7 \mathrm{~g} / \mathrm{cc}$, and thus will expand into the plastic. Targets using diamond as a tamper are now being developed. Diamond has a density of $3.0 \mathrm{~g} / \mathrm{cc}$, and thus should keep aluminum from expanding. High- $\mathrm{Z}$ materials have a much higher density, which cannot be readily matched by a low-Z tamper. Tamping with diamond may nevertheless yield a desired improvement even for high-Z target materials. Moreover, these materials start at a much higher solid density, and with compression may still achieve densities in excess of $10 \mathrm{~g} / \mathrm{cc}$, even at temperatures of 500 to $1000 \mathrm{eV}$.

\section{Diagnostics and measurement uncertainties:}

AWE has been designing and building several key diagnostics for characterizing the electron temperature, the electron density, and the target emissivity, from which the emission opacity is derived. These are:

- a medium-resolution, time-integrating (film) crystal spectrometer for measuring the absolute emissivity of the material under study.

- a medium-resolution, time-resolved (streak camera) crystal spectrometer for measuring the spectral emission of the material under study or for measuring the electron temperature and electron density.

- a pinhole camera for determining the size of the emitting region. 
The British diagnostic suite in our assessment is insufficient for highly accurate determinations of the opacities. The availability of only a single time-resolved instrument means that the opacity signal is measured in one shot and the plasma parameters in another shot. There is an inherent uncertainty in this approach because short-pulse laser shots are intrinsically variable. Moreover, this instrument represents a single-point of failure, for example, if the streak camera breaks.

The electron temperature is derived only from a single set of K-shell lines. In particular, it is determined from the ratio of the Lyman- $\alpha$ feature of H-like and of the helium- $\alpha$ feature of He-like dopants. Similarly, the electron density is derived from the broadening of these H-like and He-like lines. By using only one set of lines and from only one type of dopant, there is no mitigation of errors, including no mitigation of statistical fluctuations. Moreover, shot to shot variability of the temperature means that the pair of lines may sometimes not be the pair one would have chosen for a given temperature, i.e., the diagnostic will need to be used in a regime in which it is not reliable. We, thus, estimate that the accuracy of the temperature determination in this approach will likely be about $40 \%$, that of the density determination will be about $20 \%$.

Livermore opacity measurements on the Omega laser facility at Rochester required measurement accuracies of $5 \%$ for the electron temperature and $20 \%$ for the electron density. Compared to the Omega measurements, the emission opacity measurements on ORION fall short in the accuracy of the temperature determination. Only the density determination will likely be done with comparable accuracy.

The accuracy with which the absolute emissivities are measured is about $10 \%$, as determined in Titan and HELEN laser measurements. However, there are systematic uncertainties that need to be calibrated out, such as the frequencydependent crystal reflectivities. The latter are currently being measured for the crystals used during the Titan experiments at the Livermore FAST calibration facility, but no results are yet available.

\section{US contributed diagnostics:}

It appears clear from the above discussion that in order to meet US requirements, it will be necessary to augment the British diagnostics. US contributed diagnostics should include:

- a second medium-resolution, time-resolved crystal spectrometer for measuring the time-evolution of the electron temperature and electron density on every opacity shot using a fast streak camera. 
- a hot-electron spectrometer to assess the number of fast electrons and thus to enable the modeling of non-thermal heating and to assess the LTE-ness of the plasma target.

- a second, time-integrated, medium resolution crystal spectrometer for surveying a broad spectral region in order to derive the electron temperature from fits of multiple K-shell lines.

- a high-resolution, time-integrated crystal spectrometer for determining the spectral line shape

Among these instruments, we believe that the second time-resolved crystal spectrometer is crucial, because it will allow us to make simultaneous measurements of the electron temperature and electron density while the British instrument makes time-resolved measurements of the emissivities. In other words, having simultaneous measurements the plasma parameters while an opacity measurement is made is a critical step for obtaining reliable results.

A second, independent temperature determination is crucial for improving the accuracy of the electron temperature measurement. For this we envision an observation of multiple lines in the He-like and H-like Rydberg series. This measurement will be made in time-integrated fashion. But by observing the same key helium- $\alpha$ and Lyman- $\alpha$ lines that are also observed with the streak camera instrument, we will be able to interpolate between the time-integrated measurement to the time-resolved measurements. Using the time-resolved and the time-integrated temperature diagnostics in tandem will reduce the uncertainty in the electron temperature to about $10-20 \%$.

A further increase in the accuracy of the temperature measurement can be achieved by using two simultaneous dopants, one with a nuclear charge two or three higher than the other (e.g., aluminum together with sulfur or chlorine). This makes the diagnostic line ratio a lot more sensitive to electron temperature. Observation of the K-shell lines from the second dopant should, thus, reduce the uncertainty to about 5-10\%.

The diagnostic suite should include hot electron spectrometers for measuring highenergy electrons. A hot electron measurement on Titan provided crucial information on shot-to-shot laser performance (such as occasional pre-pulse generation) not otherwise available. If fast electrons are detected, these measurements also provide constraints on the deviation from LTE-ness of the plasma, which again is a critical ingredient for making reliable opacity measurements.

Finally, a very high-resolution crystal spectrometer trained on a small spectral region can provide line shape information, especially information of the line wings. Unlike all other instruments, which require a TIM tube for mounting, this 
instrument can reside outside the ORION target chamber, thus making it inexpensive.

Our UK collaborators have assured us that any instrument we can contribute will gladly be accommodated on ORION. Indeed, they are expecting to provide such instrumentation as part of our contribution to the collaboration.

\section{Summary:}

The advent of ORION will undoubtedly provide a platform for measuring opacities in heretofore inaccessible plasma regimes. The achievable plasma parameters will clearly be far higher than promised by any other platform. The US has the opportunity to contribute crucial temperature and density diagnostics as well as a hot electron and a spectral line shape diagnostic, and thus to ensure that the measurements will be from well diagnosed plasmas with the highest experimental certainties. The accuracy of these novel measurement can, thus, be as good as that of measurements currently obtainable from the Omega laser at much lower density and temperature.

Acknowledgements. This work was performed under the auspices of the U.S. Department of Energy by Lawrence Livermore National Laboratory under Contract DE-AC52-07NA27344. 